\title{
CGM Retrospective Data Analysis
}

\author{
Gary Scheiner, MS, CDE
}

\begin{abstract}
Continuous glucose monitoring (CGM) has increased in popularity as a daily management tool for people with diabetes and a diagnostic instrument for their healthcare providers. Achieving better clinical outcomes hinges on appropriate analysis and interpretation of data collected by CGM systems. This includes device downloading, qualification of data, and generation of applicable reports. An objectives-based analysis of the reports can yield valuable insight for fine-tuning treatment in several areas, including postprandial glucose patterns, overnight/basal stability, duration of bolus insulin action, timing of (and response to) hypoglycemic episodes, the efficacy of meal and correction insulin doses, and the impact of a variety of lifestyle activities.
\end{abstract}

\section{Introduction}

$\mathbf{I}$

F A TREE FALLS IN THE FOREST, does it make a sound? Of

course it does. But if nobody is around to hear it, what difference does it make?

The same can be said about continuous glucose monitoring (CGM) data. With modern CGM systems generating upward of 288 glucose values daily, there is no shortage of information. But if nobody analyzes it, learns from it, or adjusts to it, what's the point? Evidence suggests that retrospective pattern analysis of CGM data is one of the keys to benefitting from CGM use. ${ }^{1,2}$ This holds true whether patients are using a personal (real-time) CGM system or a professional (loaner) system. Until we have a functioning "artificial pancreas", system, retrospective analysis of CGM data by qualified healthcare providers will remain an important tool in making therapeutically beneficial decisions. The focus of this article is on obtaining CGM data, qualifying it, and interpreting it an efficient and outcome-driven manner.

\section{Obtaining the Data}

Several software programs allow clinicians to obtain key statistics and view CGM data in a graphic and customized format. Some also permit contextual evaluation by merging the CGM data with information from insulin pumps, blood glucose meters, and fitness tracking devices.

Most CGM download software focuses on presenting data in a meaningful way. However, there is a gradual movement toward offering guided assistance with the analysis of the data as well. A summary of currently available programs is provided in Table 1.

The Navigator ${ }^{\circledR}$ CGM device from Abbott (Alameda, CA) downloads to FreeStyle ${ }^{\circledR}$ Copilot software (go to www .abbottdiabetescare.com to download to your personal computer [PC]) using a Bluetooth ${ }^{\circledR}$ (Bluetooth SIG, Kirkland, WA) plug-in. Events entered into the Navigator device will appear on some reports. Data from the OmniPod ${ }^{\circledR}$ PDM (Insulet Corp., Billerica, MA) may be downloaded as well so that insulin delivery and carbohydrate data will be merged with the CGM data. FreeStyle Auto-Assist (go to www .abbottdiabetescare.com to install) uploads data from the Libre system. Although data cannot be "exported/imported" between patients and healthcare providers, users can generate PDF files of their reports and then share them via e-mail.

Dexcom $^{\circledR}$ (San Diego, CA) Studio software (go to www .dexcom.com to install) is designed exclusively for downloading and displaying data from Dexcom receivers (professional or personal versions). It is PC-based and not Web-enabled, and it does not work on $\mathrm{Mac}^{\circledR}$ (Apple, Cupertino, CA) systems. Home users may share their data by e-mail by attaching their "patient file" or PDFs of individual reports. For those who want to download Dexcom data to a Mac computer, Dexcom's Portrait on the Web can generate a series of predefined reports (go to www.dexcom.com/portrait to install).

For those using Dexcom's G5 ${ }^{\mathrm{TM}}$ system on their mobile phone, their "clarity" app generates reports from the patient's cloud-based data. Patients can easily generate a 12digit code to share with providers that provide instant access to the same reports. Healthcare providers simply go to https://clarity.dexcom.com/\#/overview to go to "clarity clinic".

Glooko (Palo Alto, CA) is a subscription service available to patients as well as clinics. It can integrate data from the Dexcom CGM as well as glucose meters and some insulin pumps. Various customized reporting options (and data sharing) are available.

Integrated Diabetes Services, Wynnewood, Pennsylvania. 
Table 1. Programs for Downloading/Analyzing Continuous Glucose Monitoring Data

\begin{tabular}{|c|c|c|c|}
\hline $\begin{array}{l}\text { CGM } \\
\text { system }\end{array}$ & Download program & Useful reports & Compatibility and use \\
\hline \multirow[t]{2}{*}{ Abbott } & CoPilot (for Navigator) & $\begin{array}{l}\text { - Glucose Modal Day } \\
\text { - Statistics } \\
\text { - Logbook }\end{array}$ & Free. PC-based, but shareable via Internet \\
\hline & $\begin{array}{l}\text { Freestyle Auto-Assist } \\
\text { (for Libre) }\end{array}$ & $\begin{array}{l}\text { - Snapshot } \\
\text { - Modal Day }\end{array}$ & Free. Mac or PC-based. Nonshareable data \\
\hline \multirow[t]{5}{*}{ Dexcom } & Dexcom Studio & $\begin{array}{l}\text { - Patterns } \\
\text { - Daily Trends } \\
\text { Glucose Trend } \\
\text { - Hourly Stats } \\
\text { - Success Report }\end{array}$ & $\begin{array}{l}\text { Free. PC-only; data file shareable via e-mail. } \\
\text { Allows sorting by day of the week }\end{array}$ \\
\hline & Portrait On The Web & $\begin{array}{l}\text { - Trend Patterns and Insights } \\
\text { - Daily Trends }\end{array}$ & Free. Mac systems only \\
\hline & Clarity App & $\begin{array}{l}\text { - Data (Trends) } \\
\text { - Data (Daily) }\end{array}$ & $\begin{array}{l}\text { Free. Web-based. Allows sorting by day of the } \\
\text { week. Utilizes ambulatory glucose profile format. }\end{array}$ \\
\hline & Glooko & $\begin{array}{l}\text { - Modal Day } \\
\text { - Calendar View }\end{array}$ & $\begin{array}{l}\text { Annual fee. Apple and Android devices supported. } \\
\text { Allows sorting by day of the week }\end{array}$ \\
\hline & Diasend & $\begin{array}{l}\text { - CGM Modal } \\
\text { - Day-by-Day } \\
\text { - Compilation }\end{array}$ & $\begin{array}{l}\text { Web-based; Mac- or PC-compatible. Requires } \\
\text { "uploader" software on computer. Easily } \\
\text { shared data. May require clinic to pay a fee }\end{array}$ \\
\hline \multirow[t]{2}{*}{ Medtronic } & CareLink & $\begin{array}{l}\text { - Sensor Daily Overlay } \\
\text { - Sensor Overlay by Meal } \\
\text { - Daily Summary } \\
\text { - Trends Summary }\end{array}$ & $\begin{array}{l}\text { Free. Web-based, PC- and Mac-compatible. } \\
\text { Data/reports easily shareable (password } \\
\text { required) }\end{array}$ \\
\hline & CareLink Pro & $\begin{array}{l}\text { - Therapy Management } \\
\text { Dashboard } \\
\text { - Episode Summary } \\
\text { - Adherence }\end{array}$ & PC-only. Intended for healthcare provider use \\
\hline
\end{tabular}

CGM, continuous glucose monitoring; PC, personal computer.

Diasend $^{\circledR}$ (Chicago, IL) (go to diasend.com to register and download the "uploader" software) is an independent Web-based program that imports and merges data from various devices (including blood glucose meters, non-Medtronic [Northridge, CA] insulin pumps, fitness trackers, and the Dexcom CGM device). It can be used with just about any computer that has Internet access. The CGM tab serves as a portal to several useful reports. The Day-By-Day reports (found under the comparison tab) display unified reports of CGM data along with information from other downloaded devices.

Medtronic offers two programs for downloading data from their real-time and professional-use CGM systems: CareLink ${ }^{\circledR}$ Personal and CareLink Pro. CareLink Personal is Web-based and accessible through both PCs and Mac computers (go to https://carelink.minimed.com to access). No software needs to be installed, but a transmission device (CareLink USB or Contour $^{\circledR}$ [Bayer Diabetes Care, Leverkusen, Germany] Next Link USB) must be used to transmit data from the device through the computer and to the Website. These devices can be obtained from Medtronic. Once downloaded, reports can be accessed by users and their healthcare providers by entering a corresponding username and passcode. For those using a sensor-integrated insulin pump, the download includes data from the pump (insulin delivery, carbohydrate entries, events) as well as the CGM data. CareLink Pro is a PC-based software package that allows healthcare professionals to see more details than are available in CareLink Personal. The Pro software detects patterns that can guide clinicians in coming to therapeutic conclusions.

\section{Qualification and Preparation}

Before evaluating a set of CGM data, it is important to qualify the information. This means verifying that the data are reliable and representative of the user's true patterns. Specifically:

1. Make sure the clock and date are set correctly on the receiver (and all other devices being downloaded).

2. Verify that sufficient calibrations were performed.

3. Ask about the conditions under which the data were collected. Were there extenuating circumstances such as stress, travel, unusual physical activity, illness, or use of steroid medications?

4. Ensure that the data are reasonably accurate. In Medtronic's CareLink Personal, take a look at the mean absolute difference percentage (MAD\%) for each full week on the Sensor Daily Overlay report. If the MAD\% is more than $15-18 \%$, the data may not be worth reviewing. On the Dexcom Daily Trend reports, observe the discrepancy between the calibration values (denoted as red diamonds) and the simultaneous sensor values (the blue dots). If there are many large discrepancies, consider passing up on the data analysis. 
Another way to ensure high-quality data and useful interpretation is to solicit the patient's involvement. When using a professional CGM device (a system worn temporarily for diagnostic purposes), it helps to have details to go along with the glucose information. Instruct the CGM device user to keep written or computerized logs of factors that influence his or her glucose levels, such as

- Timing and content of meals

- Duration and nature of exercise

- Doses of insulin and other diabetes medications

- Noteworthy events (restaurant meals, illnesses, unusual stress, pump infusion set changes, menses, etc.)

Similarly, those using personal CGM systems can help their healthcare team perform a meaningful analysis by recording key data (insulin doses, meals/snacks, exercise) for a week or two before clinic appointments.

For patients who take basal insulin by injection, a professional CGM session is a golden opportunity to evaluate the dose. Ask the patient to omit after-dinner snacks and bolus insulin for a couple of nights in order to measure the stability of the glucose level through the night. Insulin pump users can perform a series of fasts at various times of day in order to evaluate the pump's basal settings. Table 2 shows a sample basal testing schedule for insulin pump users.

\section{Data Interpretation: Statistical Summaries}

Because CGM devices generate glucose values around-theclock, the data are not skewed by checking more often during periods of high or low glucose or by only checking prior to meals. The statistics generated by CGM devices are thus more valid than those garnered from blood glucose meters.

The mean (average) sensor glucose represents a fairly true average, albeit slightly lower than reality due to a natural tendency for the systems to err on the side of lower rather than higher values, as well as the prolonged lag time that occurs when recovering from hypoglycemia. Adding 2-3\% to a CGM average is a good way to correct for these system deficiencies. A1c can then be estimated by using the eAG equation ([CGM average +46.7]/28.7). ${ }^{3}$

Another useful statistic is the standard deviation (SD). A high SD means that there are many glucose values that are significantly above or below the average. A low SD indicates a relatively low number of "outliers." From my experience, an SD that is less than $33 \%$ of the average is generally desirable.

Table 2. Sample Basal Testing Schedule FOR INSUlin PUMP USERS

\begin{tabular}{lccc}
\hline Basal test & $\begin{array}{c}\text { Eat and } \\
\text { bolus by }\end{array}$ & $\begin{array}{c}\text { Then no calories, } \\
\text { boluses, or } \\
\text { exercise until }\end{array}$ & $\begin{array}{c}\text { Evaluate } \\
\text { sensor data }\end{array}$ \\
\hline Overnight & 6 p.m. & $\begin{array}{c}7 \text { a.m. (no night } \\
\text { snacks) }\end{array}$ & 10 p.m.-7 a.m. \\
Morning & 11 p.m. & $\begin{array}{c}12 \text { noon (skip } \\
\text { breakfast) }\end{array}$ & 7 a.m.-12 noon \\
Afternoon & 8 a.m. & $\begin{array}{c}5 \text { p.m. (skip } \\
\text { lunch) }\end{array}$ & 12 noon-5 p.m. \\
Evening & 1 p.m. & $\begin{array}{c}10 \text { p.m. (late } \\
\text { dinner) }\end{array}$ & 5 p.m. -10 p.m.
\end{tabular}

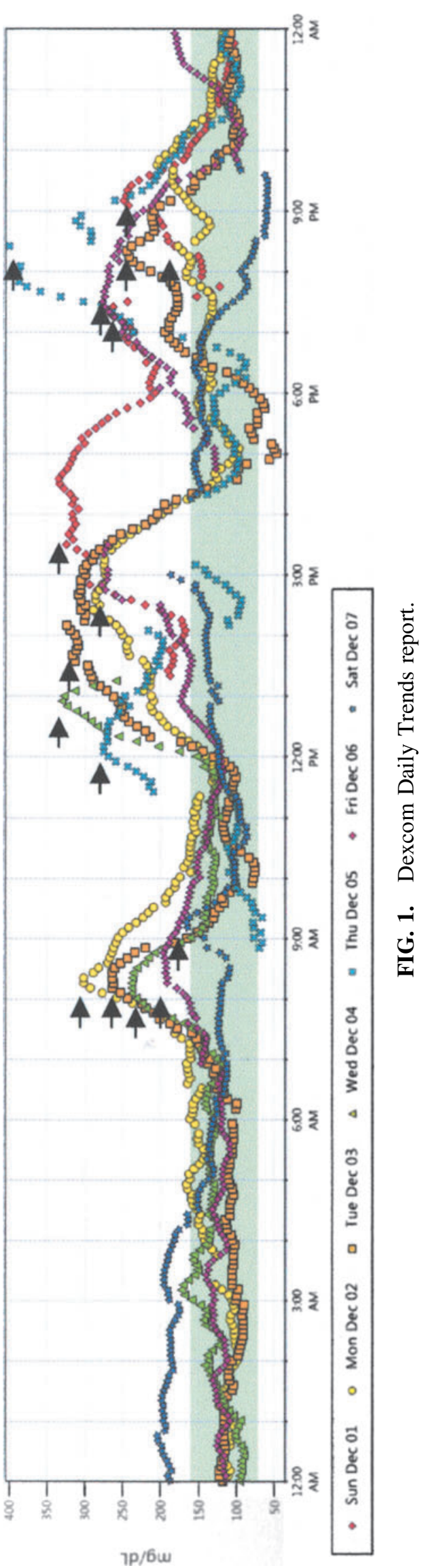


Overlay by Meal Event (mg/dL)

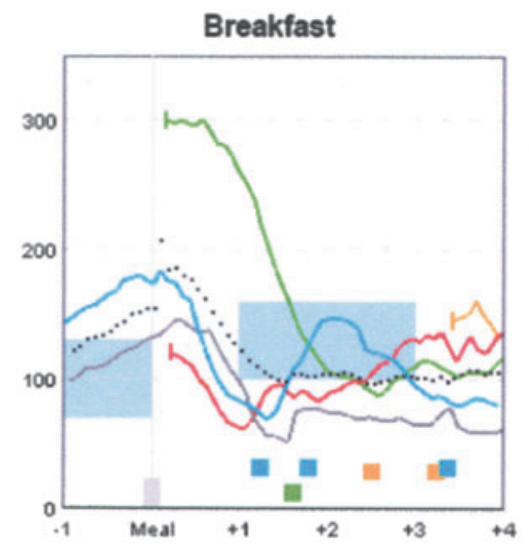

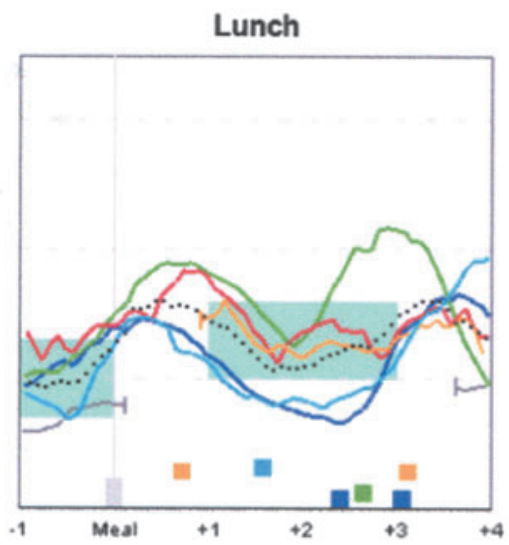

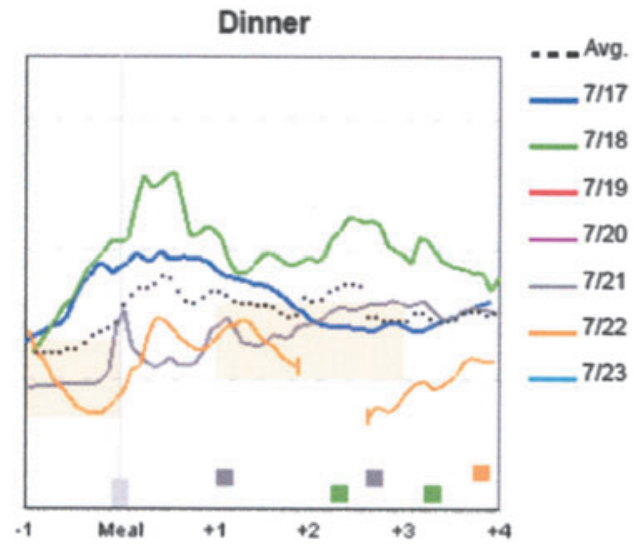

FIG. 2. Medtronic Sensor Overlay by Meal report. Avg, average.

An SD that is more than $50 \%$ of the average indicates excessive variability. Because glucose variability may be associated with both risk of long-term complications and hindering shortterm quality of life, efforts to minimize, measure, and manage glycemic excursions are worthwhile in addition to management of hemoglobin A1c. ${ }^{4}$

A highly practical statistic is the percentage of time spent above, below, and within one's target glucose range. The target range can be customized on each software program and should be individualized for each patient. A major goal of diabetes treatment is to spend as much time as possible within the target range and as little time above and below target. In our practice we endeavor to attain $70 \%$ or more time in range and less than 5\% of time below range for most of our clients...recognizing that this may take time to achieve. Measuring time in range can be useful for evaluating therapeutic changes and motivating patients to continue with behaviors that are producing desired results.

\section{Data Interpretation: Trend Graph/Pattern Analysis}

These are the "money reports" for which CGM devices are famous. They are also the source of anxiety for many clinicians. As useful as trend graphs are, they can also be quite confusing and time consuming to study. That's why it is important to have a solid game plan. Rather than going into the graphic reports with an open mind, set yourself up with a solid agenda.

There are several valuable insights to derive from CGM trend graphs. My "top 10" include the following:

1. Assessing the magnitude (and timing) of postprandial glucose peaks

2. Determining the effectiveness of (or need for) mealtime insulin doses

3. Quantifying the correction factor/insulin sensitivity

4. Verifying that basal insulin doses are set properly

5. Measuring the duration of the insulin action curve

6. Uncovering patterns of hypoglycemia
9
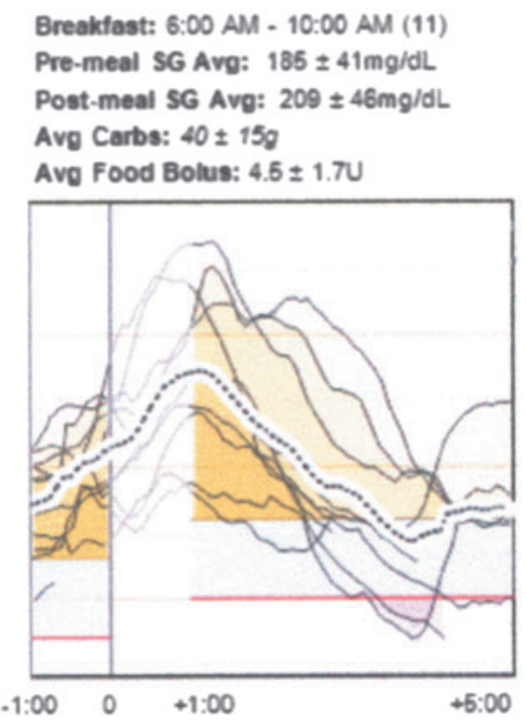

9

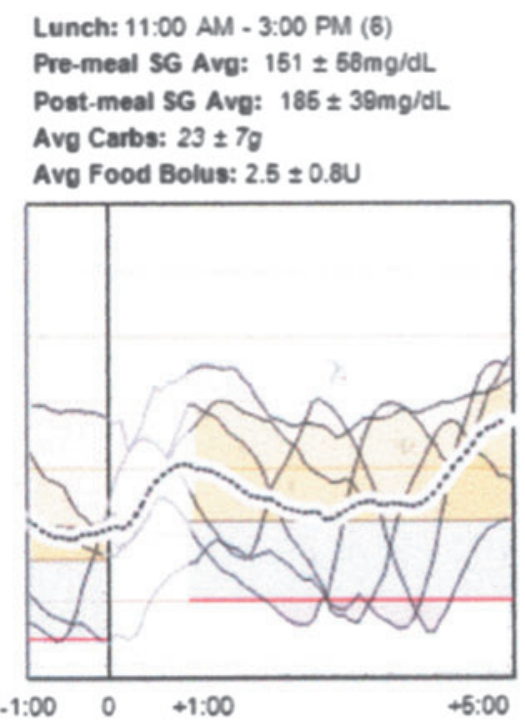

7

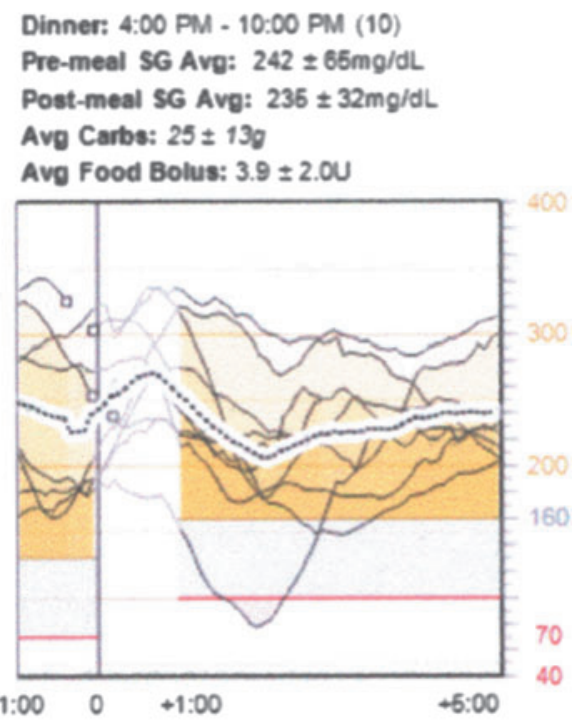

FIG. 3. Medtronic Therapy Management Dashboard report. Avg, average; Carbs, carbohydrates; SG, serum glucose. 

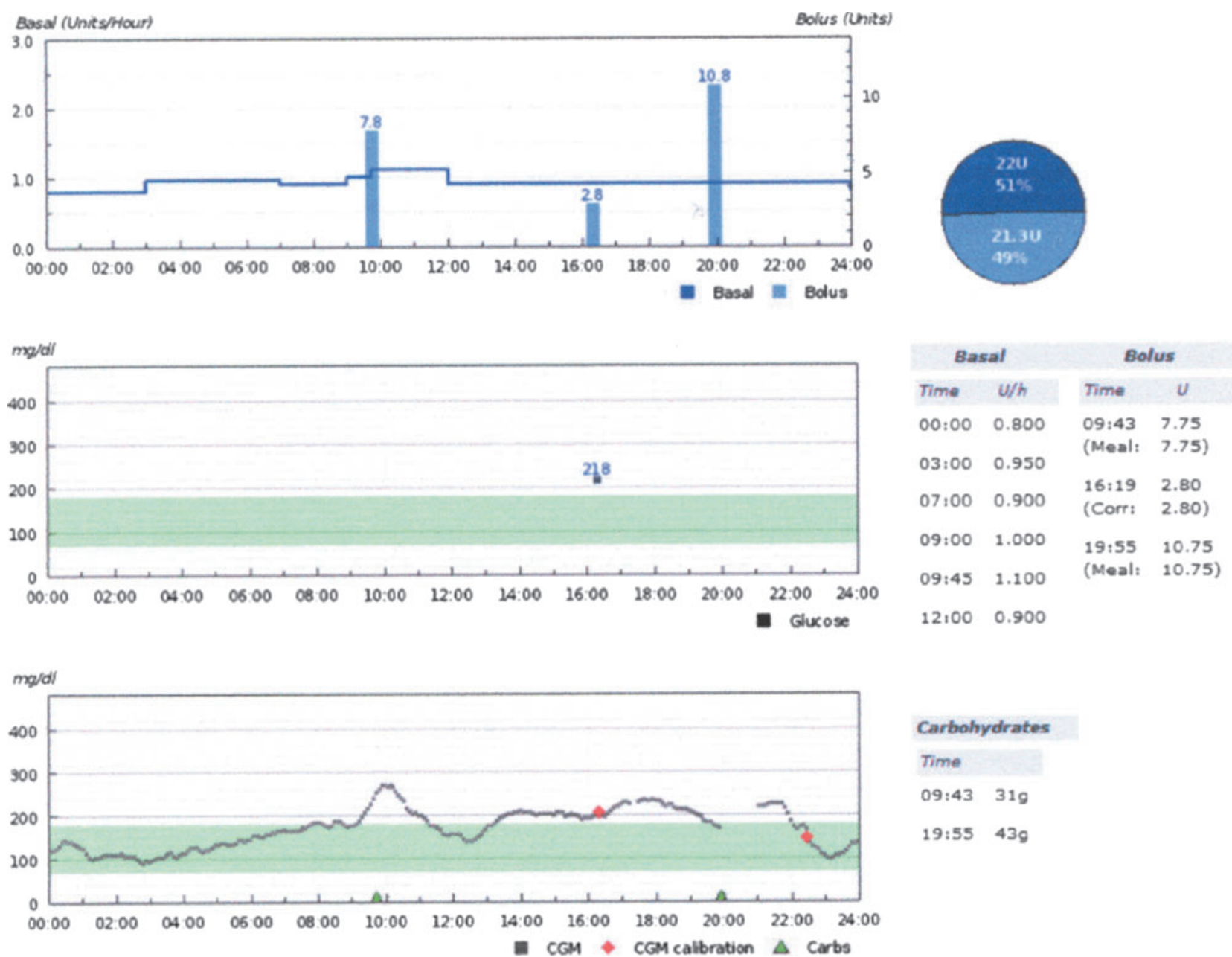

FIG. 4. Diasend Day-by-Day comparison report. Carbs, carbohydrates; CGM, continuous glucose monitoring.

7. Evaluating the treatment of hypoglycemia

8. Titrating glucose-lowering medications

9. Discovering the impact of lifestyle events and activities

10. Revealing system abuses or behaviors that may be sabotaging one's control

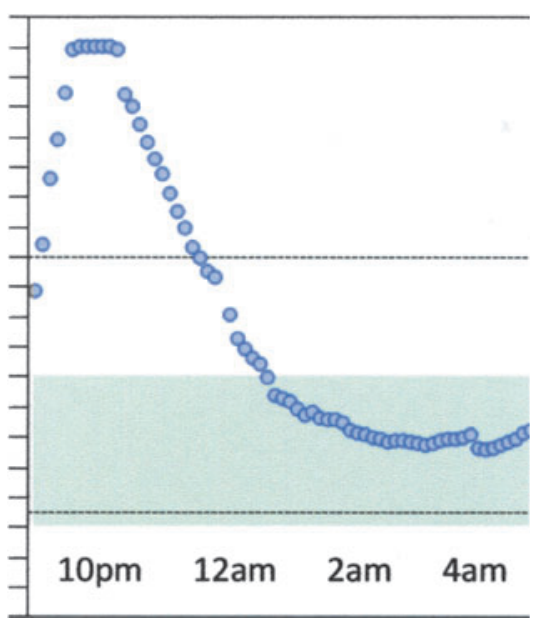

FIG. 5. Dexcom Glucose Trend graph showing duration of insulin action.

\section{Postprandial assessment}

The only thing harder than getting patients to check their blood sugar before each meal is getting them to check after meals. Besides, there is no telling exactly when the blood glucose level will hit its apex following a given meal. Postprandial peaks appear on the CGM trend graphs (and downloaded reports) no matter when they take place. In most cases, it is relatively easy to spot the postmeal peaks in a trend graph report. To be certain, have your patients use the event markers in their CGM device, or match the sensor graph with bolus/carbohydrate data from their insulin pump. In Figure 1 postprandial peaks have been marked with arrows on a Dexcom Daily Trends report.

In Figure 2 glucose values following a week's worth of meal boluses appear in a Sensor Overlay By Meal report from Medtronic's CareLink Personal program.

\section{Mealtime coverage}

For those who take mealtime insulin, observing the glucose pattern over the following 3-4 h provides a good assessment of the dose. For those who do not take mealtime insulin, observing the postmeal pattern may indicate the need to switch/add glucose-lowering medications or initiate mealtime insulin dosing. In Figure 3 the Therapy Management 
Dashboard (from CareLink Pro) indicates low glucose values following lunch (less insulin needed at lunch) and highs following dinner (more needed at dinner).

\section{Correction Dose Analysis}

In most cases an individual's correction factor (or insulin sensitivity) is inversely related to the total amount of insulin taken per day. ${ }^{5-7}$ Various formulaic approaches can be used to estimate the correction factor, but the formulas don't work for everyone. In addition, many people find that their sensitivity to insulin varies by time of day. For these reasons, it is prudent to verify correction factors empirically based on sensor tracings. When a correction bolus is given without a concurrent meal dose (and no food is consumed and no more bolus insulin given) for several hours, the resultant glucose level should indicate whether the correction factor is set properly. Of course, it is necessary to verify basal insulin doses before testing the correction formulas because an incorrect basal setting will influence the outcome of a correction dose.

\section{Basal insulin fine-tuning}

CGM is an excellent tool for fine-tuning basal insulin doses. Even when accuracy is in question, CGM's ability to detect rising and falling glucose levels is unparalleled. Because basal insulin's role is to achieve stability in glucose levels in the absence of digesting food, exercise, and active mealtime insulin, any significant rise or fall in a fasting/ inactive state indicates a need to adjust the basal insulin dose. In Figure 4, taken from a Diasend Day-By-Day Comparison report, a glucose rise between 3 a.m. and 9 a.m. (with no food digesting) indicates a need for a basal rate increase.

\section{Measuring the duration of insulin action}

Because all modern insulin pumps deduct bolus insulinon-board (or "active insulin") from correction and/or meal boluses, knowing one's true duration of bolus action will ensure more accurate dosing and better control. Insulin action curves vary from person to person based on metabolic rate and subcutaneous absorption patterns. There is evidence that very large doses tend to have a longer action curve than smaller ones ${ }^{8}$ and that those with an accelerated metabolism may have a shorter action curve. Underestimation of the insulin action curve will lead to chronic underestimation of insulin-on-board and sets a pump user up for frequent overdosing and hypoglycemia. Overestimation may lead to chronic underdosing of insulin and frequent hyperglycemia.

To determine the duration of bolus action, observe the glucose pattern that follows a fairly typical correction dose, with no food eaten. Once again, it is best to verify basal insulin doses before examining the duration of bolus action. Figure 5 features a Dexcom glucose trend graph indicating a 4-h duration of insulin action (with a correction bolus delivered at 10:30 p.m., glucose ceases to drop at 2:30 a.m.).

\section{Discovering patterns of hypoglycemia}

CGM offers a glimpse into what is taking place when patients are asleep or going for prolonged intervals between fingerstick glucose measurements. In particular, overnight

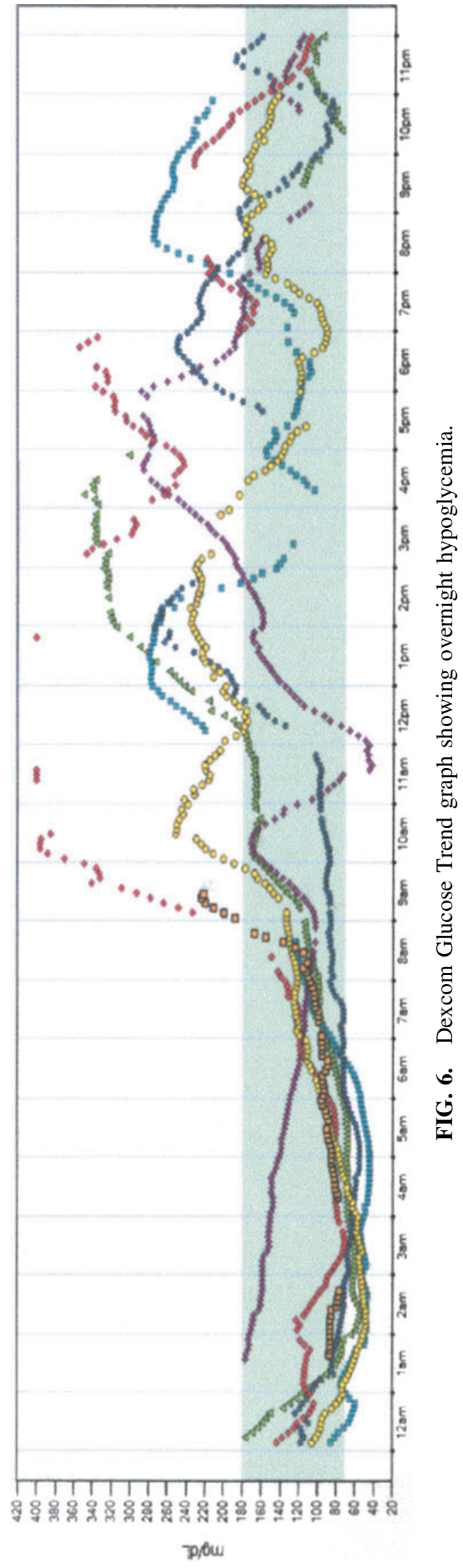


patterns may reveal rebounds from asymptomatic lows that result in fasting highs and/or insulin resistance in the morning. For example, the Glucose Trend Graph in Figure 6 indicates middle-of-the-night lows producing rebound highs the following morning.

\section{Evaluating the treatment of hypoglycemia}

Given that most peoples' primal urge is to eat and continue to eat until the symptoms of hypoglycemia disappear, it is very common to see inappropriate and excessive treatment of low blood sugar. (Overtreatment can be exacerbated by prolonged lag time that exists in CGM systems when in a state of hypoglycemia.) For others, hypoglycemia is ignored or undertreated in order to avoid unwanted calories. A quick look at CGM reports can provide insight into what happens after hypoglycemic events. Figure 7 features a CareLink Pro Episode Summary Report indicating the frequency with which hyperglycemia was preceded by hypoglycemia.

\section{Titrating medications}

When changing or adding diabetes medications, it is helpful to know when a therapeutically effective dose has been achieved. Daily trend graphs reveal glycemic changes almost immediately. This can be particularly helpful for avoiding overdosing on medications that can cause nausea or hypoglycemia, as well as evaluating the effects of different combinations of oral and injectable glucose-lowering medications.

\section{Discovering the impact of lifestyle events and activities}

This may be considered a shared responsibility between patient and clinician. It would certainly be nice if all patients took notes and made their own observations based on realtime CGM data, but this is not usually the case. Clinicians are the ones who usually point out cause-and-effect relationships with the aid of patients' notes and/or event markers in the CGM system itself.

For example, individual day trend graphs can show the impact of:
- Various forms of exercise

- Unusual stress

- Specific food types, such as restaurant meals

- Alcohol consumption

Multiple-day trend graphs, day-of-the-week reports, and long-term patterns can reveal the effects of:

- Certain types of illnesses

- Steroid medications or injections

- Menstrual cycles

- Work/school versus off days

- Pump infusion device changes

For example, Figure 8 shows elevated nighttime glucose levels on weekends (the result of take-out/restaurant food and late-night snacking on Friday and Saturday nights). Figure 9 shows well-managed overnight glucose levels on weeknights for the same patient during the same 2 weeks.

\section{Revealing system abuses or behaviors that may be sabotaging one's control}

Patients can sometimes undermine a provider's best efforts to improve glycemic control. Remember, people with diabetes are still people, so we can expect the occasional malfeasance. By combining CGM reports with data from pumps, smartphone applications, and written logs, the sources of undesirable glucose patterns can sometimes be uncovered.

Medtronic's Episode Summary Reports in its CareLink Pro software can serve as a goldmine of hidden secrets. Check the events preceding both high and low glucose alerts... what you find may surprise you. Delayed infusion set changes, overriding the pump's dosage recommendations, ignoring rise/fall rate alerts, and many other sources of dysglycemia can be found in these reports.

Likewise, any sensor trend graph report can reveal missed mealtime insulin doses (just look for substantial, unexplained rises around mealtimes), late boluses (very sharp rises followed by very sharp drops), and misrepresentation of written glucose logbooks (sensor data don't lie!).

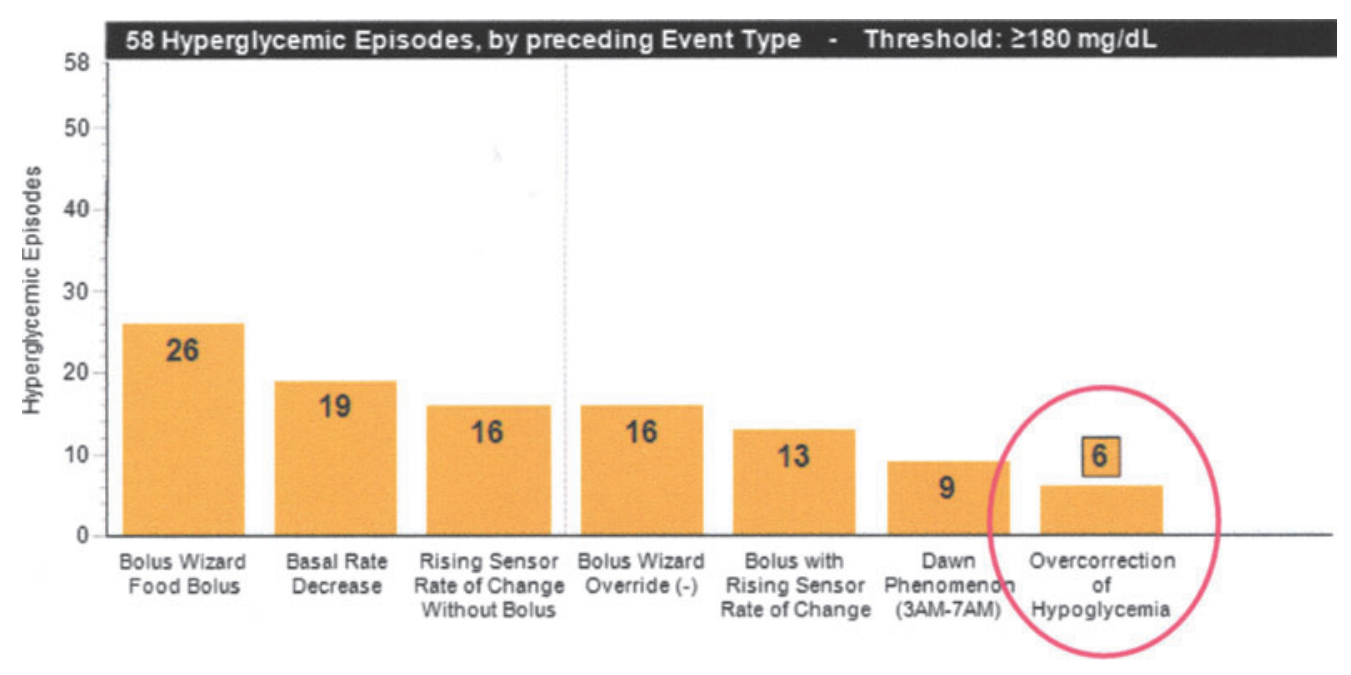

FIG. 7. Medtronic Episode Summary report. 

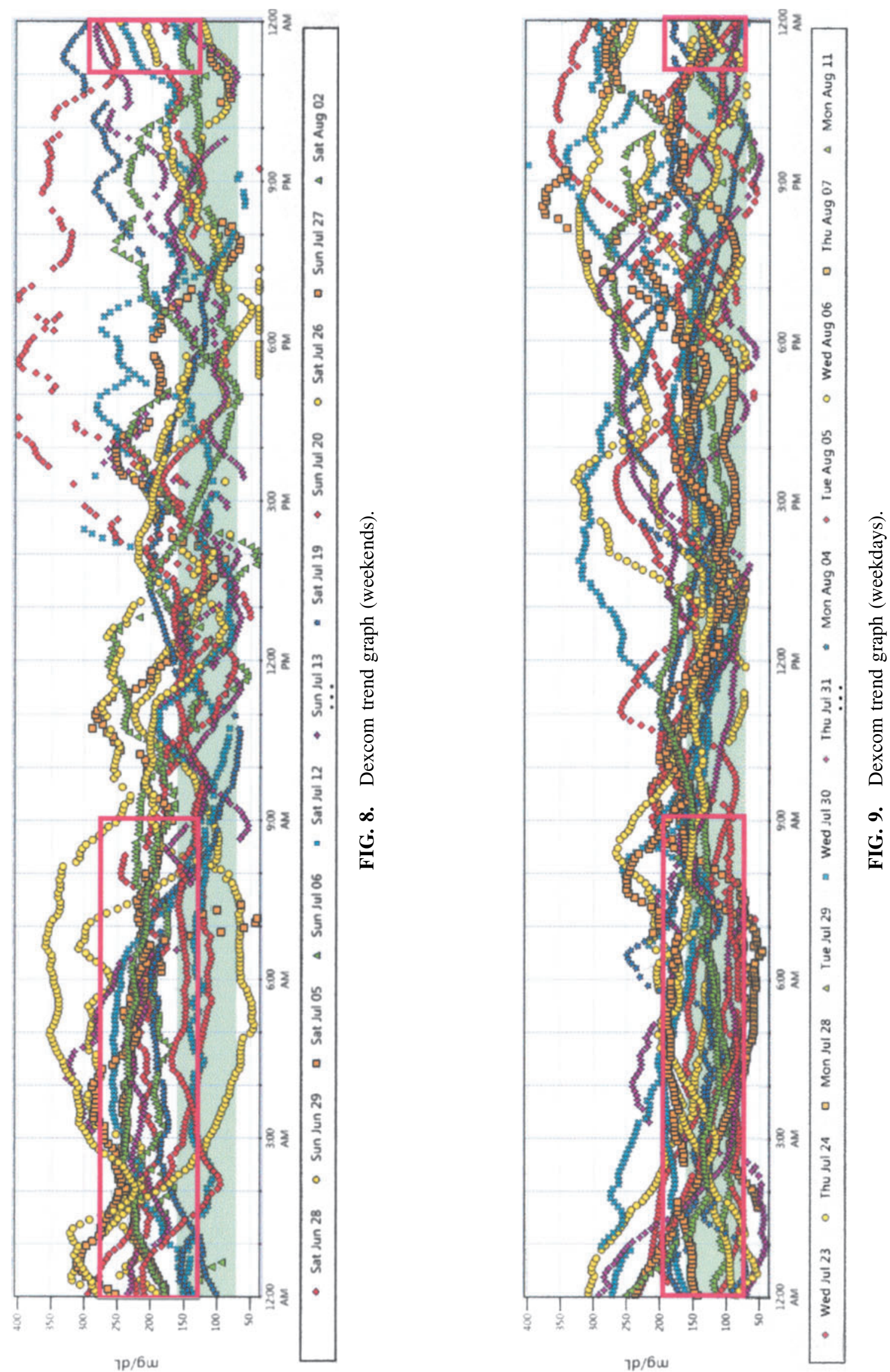


\section{Take Manageable Bytes}

Clearly, there is a great deal that can be learned from CGM data. Before you come down with a bad case of "datus overwhelmus," don't forget that all of this is not meant to be covered in a single patient visit. Try to glean a few insights at a time. For example, the first time you look at your patient's reports, you might just want to focus on his or her basal insulin settings and perhaps look for patterns of hypoglycemia that require an immediate adjustment. At the next appointment, focus on the effectiveness of mealtime doses and try to evaluate the duration of insulin action, and so on. Regardless of the topics addressed, it is worthwhile to download and analyze CGM data at each office visit.

Of course, if a particular factor (such as a new medication or exercise) plays a major role at the time of the visit, that topic should take top priority. Fixing everything that might be wrong with a diabetes management program takes time, insight, and effective technology. Steady progress is what you're after. That's what will keep your patients engaged, satisfied, and coming back for more.

\section{Limits to Analysis}

Although there is virtually no limit to the amount of data available, there are limits to what can be done with it. Interpatient variability makes it very difficult to standardize how we look at patient data. Individualization remains critical in performing effective analysis and developing safe and appropriate management strategies.

The dearth of research on the analysis of CGM data also limits our ability to improve clinical outcomes. The observations and conclusions drawn in this article are merely the result of my own personal experience. Although we have managed to achieve positive outcomes in our patient population as a whole, the healthcare community would benefit greatly from evidence-based clinical research, and additional case studies on this topic.

\section{Further Reading}

For additional information on this topic, the reader is directed to Kaufman, ${ }^{9}$ Scheiner, ${ }^{10}$ and Chase and Messer. ${ }^{11}$

\section{Author Disclosure Statement}

No competing financial interests exist.

\section{References}

1. Pearson I, Bergenstal R: Fine-tuning control: pattern management versus supplementation. Diabetes Spectrum 2001; 14:76.

2. Ritholz M, Atakov-Castillo A, Beste M, et al.: Psychosocial factors associated with use of continuous glucose monitoring. Diabet Med 2010;27:1060-1065.

3. Nathan D, Kuenen J, Borg R, et al.: Translating the A1C assay into estimated average glucose values. Diabetes Care 2008;31:1473-1478.

4. Hirsch I, Brownlee M: Beyond hemoglobin A1c-need for additional markers of risk for diabetic microvascular complications. JAMA 2010;303:2291-2291.

5. King A: Continuous glucose monitoring-guided insulin dosing in pump-treated patients with type 1 diabetes: a clinical guide. J Diabetes Sci Technol 2012;6:191-203.

6. Hagnas R: Type 1 Diabetes-A Guide for Children, Adolescents, Young Adults and Their Caregivers. New York: Marlowe \& Company, 2005.

7. King A, Armstrong D: A prospective evaluation of insulin dosing recommendations in patients with type 1 diabetes at near normal glucose control: bolus dosing. J Diabetes Sci Technol 2007;1:42-46.

8. Gagnon-Auger M, du Souich P, Baillargeon JP, et al.: Dosedependent delay of the hypoglycemic effect of short-acting insulin analogs in obese subjects with type 2 diabetes-a pharmacokinetic and pharmacodynamic study. Diabetes Care 2010;33:2502-2507.

9. Kaufman FR: Insulin Pumps and Continuous Glucose Monitoring: A User's Guide to Effective Diabetes Management. Arlington, VA: American Diabetes Association, 2012.

10. Scheiner G: Practical CGM-A Guide to Improving Outcomes Through Continuous Glucose Monitoring. Arlington, VA: American Diabetes Association, 2015.

11. Chase HP, Messer L: Understanding Insulin Pumps \& Continuous Glucose Monitors, 2nd ed. Denver: Children's Diabetes Foundation, 2010.

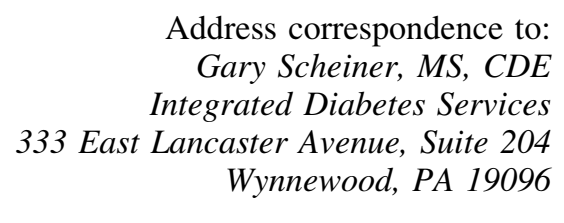

E-mail: gary@integrateddiabetes.com 